\section{Selenium-Homotaurocholsäure- Retentionstest}

\author{
A. M. Gressner ${ }^{1}$ und O. A. Gressner ${ }^{2}$ \\ ${ }^{1}$ Labor Dr. Wisplinghoff Berlin, Berlin, Deutschland \\ ${ }^{2}$ Labor Dr. Wisplinghoff Köln, Köln, Deutschland
}

\section{Synonym(e) ${ }^{75}$ SeHCAT-Test}

Definition Der zur Diagnostik der Gallensäuremalabsorption eingesetzte nuklearmedizinische Funktionstest beruht auf der oralen Aufnahme der ${ }^{75}$ Selenium- $\left({ }^{75} \mathrm{Se}\right.$-)markierten konjugierten Gallensäure Homotaurocholsäure, die im terminalen Ileum aktiv resorbiert und mit der $>$ Galle sezerniert wird, deren Menge an festgelegten Zeitpunkten mit einem Ganzkörper-Counter oder einer Gamma-Kamera quantitativ bestimmt wird.

Beschreibung Die synthetische, in Position 24-C mit ${ }^{75} \triangleright$ Selen-markierte konjugierte Gallensäure ( $\vee$ Gallensäuren) wird nach Ermittlung des Nüchternnullwerts oral appliziert ( $37 \mathrm{kBq}$ bzw. $10 \mu \mathrm{Ci}$ ). Nach 6 Stunden wird die Ausgangsaktivität über dem Abdomen mit einer Gamma-Kamera bestimmt. Weitere Messungen erfolgen an den nachfolgenden Tagen (Tage 1, 2, 4 und 7). Die radioaktive Gallensäure wird aktiv im Ileum resorbiert und durchläuft etwa drei- bis zwölfmal pro Tag den enterohepatischen Kreislauf. Die Gallensäureausscheidung wird somit mehrfach reproduziert. Eine Quantifizierung ihrer Ausscheidung im Stuhl ist nicht notwendig.

Retentionsnormwerte in der Gallenblase sind in der folgenden Tabelle zusammengefasst:

\begin{tabular}{|l|l|}
\hline Zeitpunkt (Tag) & Retention (\%) \\
\hline 1 & $>80$ \\
\hline 2 & $>65$ \\
3 & $>50$ \\
\hline 7 & $>19$ \\
\hline
\end{tabular}

Der ${ }^{75}$ SeHCAT-Test stellt ein sehr sensitives Verfahren zur Diagnostik ( $\triangleright$ Sensitivität, diagnostische) einer Gallensäuremalabsorption, z. B. bei Ileumresektionen von $20 \mathrm{~cm}$, dar und ist dem ${ }^{13} \mathrm{C}$-Glykocholat-Atemtest überlegen.

\section{Literatur}

Stein J, Wehrmann T (Hrsg) (2006) Funktionsdiagnostik in der Gastroenterologie. 2., vollst. überarb. und erweit. Aufl., Springer Medizin Verlag, Berlin/Heidelberg/New York 